\title{
Progress in periodically oriented III-nitride materials
}

\author{
Jennifer Hite \\ US Naval Research Laboratory
}

The ability to grow III-nitride structures with alternating c-plane orientation has garnered interest in using these materials for new application spaces, such as frequency conversion. An overview of recent progress in growing periodically oriented (PO) III-nitrides is discussed, including AIN, AIGaN, and GaN. Successes in fabricating thick PO GaN structures (>500mm) for uses in frequency conversion are highlighted.

Keywords B1. Nitrides, B2. Semiconducting III-nitride materials, A1. Crystal structure, B2. Nonlinear optic materials, A1. Polarity

\section{Introduction}

The family of III-nitride materials is well known for applications in electrical and optoelectronic devices. Some of the more common applications include transistors and visible LEDs, along with a push towards using Al-containing alloys for DUV LEDs [1-7]. However, all of these structures are based on using a single crystal orientation. Engineering structures with opposing polar faces (III- or N-polar) abutting one another on a single substrate opens a promising avenue to using these materials for frequency conversion via quasi-phase matching [8-10]. The interest in III-Ns in frequency conversion over more conventional materials stems from their wide band gaps, high thermal conductivity, and wide transparency windows [11].

On sapphire substrates, creating these engineered structures takes advantage of the ability to control the polar orientation of III-nitrides by manipulation of the buffer layer. Growth of GaN, AIN, and alloys of the two on sapphire substrates are generally III-polar when grown on low temperature AIN buffer layers of sufficient thickness $(>15 \mathrm{~nm})$, while those on sapphire substrates without a buffer layer are N-polar [12-14]. The process to create periodically oriented (PO) structures, which consist of alternating regions of opposing crystal orientations in the c-direction consists of several steps. First, the buffer layer, or buffer layer plus III-polar nitride layer, is grown. It is then patterned and etched back to the sapphire substrate.

Afterwards, III-N layers are grown, with N-polar material grown over the regions of revealed substrate while simultaneously growing III-polar material over the areas with the buffer layer. This succession is shown in Figure 1. This has successfully been demonstrated with GaN, AlGaN, and AIN [15-17].

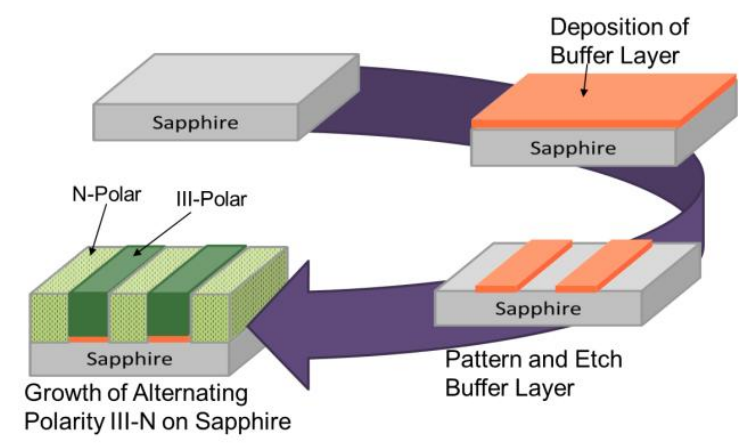


Figure 1. Process schematic used to create periodically oriented III-nitride materials. First, a buffer, or inversion, layer is grown or deposited. This layer is patterned and etched back to the sapphire substrate. A III-N layer is deposited, resulting in III-polar material over the buffer layer and N-polar over the bare sapphire.

Although this process on sapphire substrates is useful in most thin film nitride device structures and could be employed in waveguide structures for frequency conversion, to achieve bulk frequency conversion equivalent to commercially available materials, thick layers of IIInitrides, on the order of millimeter thicknesses, are required. This is because thin films on sapphire are strained and have a large density of dislocations. Thick layers also reduce internal reflection and scattering. To reach these thicknesses, native substrates are required to allow for thick growth with high crystal quality. The most successful method of creating thick PO GaN structures on $\mathrm{N}$-polar substrates has subtle differences from the methods on sapphire, namely in the use of a deposition of patterned oxide to selectively grow the inversion layer. Although other inversion layers, such as Mg-doping have been demonstrated, they have issues in interface quality or require large concentrations of $\mathrm{Mg}$, which can lead to uncontrolled inversion domains and clustering $[18,19]$. The lift-off oxide approach results in a patterned inversion layer, but maintains an epi-ready surface without plasma etch-induced damage [2022] prior to regrowth [23-25].

\section{Al-Containing Nitrides}

Recently, PO AIN structures have been grown on sapphire substrates via metal organic chemical vapor deposition (MOCVD) $[17,26]$. These structures showed a well-defined boundary between the Al- and $\mathrm{N}$ - polar regions with very little difference in growth rate between the two. The latter is very different than what has been observed with PO GaN growth [13,27]. In this case, the $\mathrm{N}$-polar regions also showed columnar growth in the initial nucleation on sapphire and is associated with rougher growth.

Some of the first PO AIGaN structures reported were thin GaN/AIGaN/GaN structures grown on sapphire by a hybrid molecular beam epitaxy (MBE) and MOCVD approach, and used Al concentrations between 15 and 30\% [15]. This showed the feasibility of creating such structures. A more recent study shows PO AIGaN stripes with much higher Al content (60-100\%) [16]. Both PO AIN and PO AIGaN could be useful for DUV applications.

\section{Progress in GaN}

Although PO $\mathrm{Al}_{\mathrm{x}} \mathrm{Ga}_{1-\mathrm{x}} \mathrm{N}$ structures are beginning to be investigated, $\mathrm{PO}$ GaN structures on sapphire have been more rigorously examined, with an eye towards both frequency conversion as well as more conventional electrical device structures.

\subsection{PO GaN on Sapphire Substrates}

Using the method described in the introduction, several groups have reported PO GaN structures on sapphire substrates using MBE and MOCVD [12,13,29-31]. As in the case for PO AIGaN structures, the N-polar regions are generally rougher than the Ga-polar regions [32]. From shifts in the $A_{1}(L O)$ peak, micro-Raman spectroscopy showed an elevated free-carrier concentration in the $\mathrm{N}$-polar region, which indicates higher impurity incorporation of the $\mathrm{N}$ - 
polar face over the Ga-polar face even when grown simultaneously [33]. This is a trend that has been shown in blanket film growth as well [34]. This study also showed a slight shift in the Raman $E_{2}$ peak with polarity, indicative of slightly larger compressive in-plane stress for the Gapolar material [33]. Work function studies have shown a difference of $0.2-0.25 \mathrm{eV}$ between the two polar regions [35,36].

One interesting aspect of these PO structures is the inversion domain boundary (IDB) that occurs at the junction of the two polar regions. In examining these structures using photoluminescence (PL) spectroscopy, an intense recombination was observed at the IDB $[13,32,37]$. In PL, it has been shown to be an order of magnitude higher in intensity and 30-40 meV lower in energy than bulk GaN, as well as being polarized parallel to the boundary [37]. Temperature dependent PL studies correlate with calculations by Fiorentini [38] indicating the emission arises from localized potential maximum in the IDB coupled with minima in the surrounding polar regions [32]. This intense emission was also observed in UV photoelectron emission microscopy (PEEM) [35]. In transmission electron microscopy (TEM) images, scanning electron microscopy (SEM) images, etching studies, and work function investigations, the IDB has been shown to range laterally in thickness from $50 \mathrm{~nm}$ to $5 \mu \mathrm{m}$ and have mixed polarity $[12,36,37]$. This mixed polarity region has been attributed to imprecise etching of the AIN buffer layer and could contribute to a non-vertical IDB [32,37]. However, the PEEM study indicated that the IDB could exhibit rectifying behavior for electronic transport across the IDB [34].

Testing out this indication, several device structures have been fabricated, both electrical and optical. The electrical structures have included $\mathrm{p} / \mathrm{n}$ junctions and a MESFET $[31,40,41]$. These demonstrate the feasibility of using the IDB as a lateral junction. For optical devices, there was a single study designing and growing PO GaN stripes for wavelength conversion [8]. Though the reported single harmonic generation was low in efficiency, it also demonstrated the possibilities for this type of structure.

\subsection{PO GaN on Bulk Substrates}

Although several methods to control GaN polarity on GaN have been reported [42-45], this section will focus on the effort that have resulted in thick PO GaN structures using a low temperature AIN inversion layer on N-polar GaN substrates $[23,24]$. TEM images show the IDB to be extremely sharp and vertical (Figure 2). Along with these TEM results, etching studies and SEM images have shown a very distinct boundary between polar regions without indications of mixed polarity at the boundary. With careful optimization of growth conditions, the growth rates of the two polar materials can be nearly matched, with height differences under $80 \mathrm{~nm}$ [27]. PO GaN templates up to $2 \mu \mathrm{m}$ in thickness have been grown by MOCVD on bulk GaN substrates. For these thin layers, a large difference in dislocation density has been observed in the two polarities. Low densities are observed in the homoepitaxially grown N-polar regions, matching that of the substrate. The Ga-polar regions show higher dislocation densities, as they are grown heteroepitaxially over the inversion layer [23]. Scanning Kelvin probe microscopy (SKPM) on theses samples have shown a $0.3 \mathrm{eV}$ difference in surface potential, similar to that seen on PO GaN structures on sapphire [46]. The study also indicated that the native oxide on the $\mathrm{N}$-polar regions was better at charge trapping than that over the Ga-polar regions. 


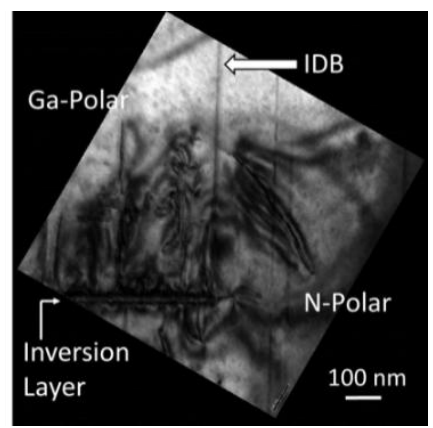

Figure 2. TEM cross-section of PO GaN structure on an N-polar GaN template. New dislocations initiating at inversion layer interface in Ga-polar region can be observed.

To produce thick PO GaN structures, $2 \mu \mathrm{m}$ thick MOCVD-grown PO GaN structures have been used as templates for additional hydride vapor phase epitaxy (HVPE) growth. In order to produce thick PO GaN structures, it is imperative to reduce differences in the growth rates of the two polarities. If not equalized, these differences result in a saw-tooth or picket-fence type of morphology at the surface, as seen in Figure 3. This can lead to nucleation of polycrystalline material, which rapidly dominates the growth, ruining the structures. The saw-tooth pattern can also affect impurity incorporation, with low impurity incorporation on +c-oriented facets, higher incorporation on -c-oriented facets, and even higher incorporation on semi-polar facets [34]. Even with differences in growth rate, TEM and SEM images show that the IDB remains vertical throughout the PO structure.

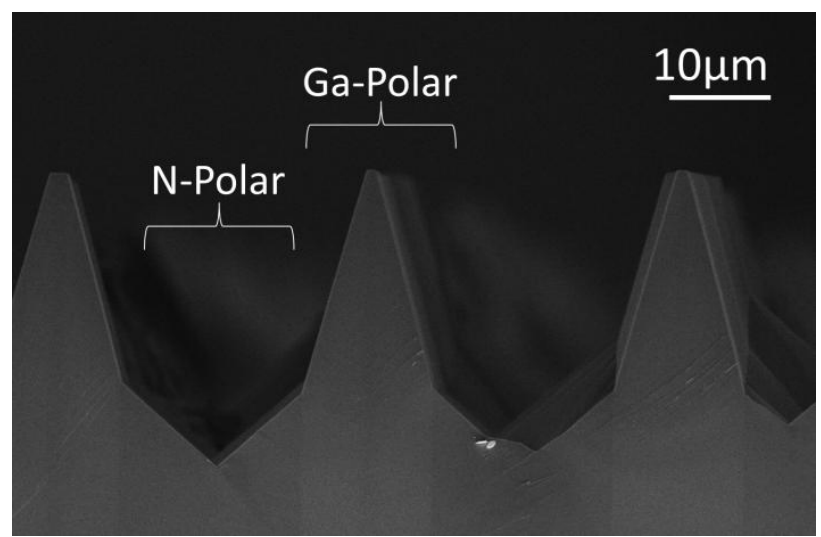

Figure 3. Cross-sectional SEM image of the saw-tooth pattern at the surface of a thick (80 $\mu \mathrm{m})$ PO GaN HVPE extension on a $2 \mu \mathrm{m}$ thick MOCVD-grown PO GaN template on a bulk HVPE substrate. This is formed due to differences in growth rate between the two polar regions.

Overcoming these troubles, PO GaN structures consisting of $16 \mu \mathrm{m}$ wide stripes have been produced with thicknesses reaching $1 \mathrm{~mm}$. To date, structures with thicknesses of 100 , 200, and $500 \mu \mathrm{m}$ have been optically measured for frequency conversion [47]. These samples show second harmonic generation with a strong peak with a width of $10 \mathrm{~nm}$, using a pump wavelength of $1.6 \mu \mathrm{m}$. The peak position variation between samples was $\pm 1 \%$. Calculations show that the efficiency of these samples is $40 \%$ of the ideal structure. The efficiency was 
reduced by large variations in free carrier concentration as well as errors in the period of the structure. Further optimization in growth and processing should increase the efficiency.

\section{Summary}

In summary, PO III-nitride structures of AIN, AIGaN, and GaN have been grown. The Alcontaining PO structures are still being developed for device applications. Thick PO GaN structures have been produced that show single harmonic generation. This research offers the promise of engineered materials with custom lateral and vertical polarity variations for applications in novel electronic and optoelectronic devices, a subset of which are expected to be suitable for non-linear optics.

\section{Acknowledgements}

This work was supported under the Office of Naval Research.

\section{References}

1. Lidow, A., Strydom, J., de Rooij, M. \& Reusch, D., GaN Transistors for Efficient Power Conversion, 2nd ed. (John Wiley \& Sons Ltd., West Sussex, United Kingdom, 2015).

2. Anderson, T. J. et al., An AIN/Ultrathin AIGaN/GaN HEMT Structure for Enhancement-Mode Operation Using Selective Etching. IEEE Electron Device Letters 30 (12), 1251-1253 (2009).

3. Kanamura, M. et al., Enhancement-Mode GaN MIS-HEMTs With n-GaN/i-AIN/n-GaN Triple Cap Layer and High- k Gate Dielectrics. IEEE Electron Device Letters 31 (3), 189-191 (2010).

4. Chang, C. -Y. et al., Reverse gate bias-induced degradation of AIGaN/GaN high electron mobility transistors. Journal of Vacuum Science \& Technology B 28, 1044-1047 (2010).

5. Meneghini, M., Tazzoli, A., Mura, G., Meneghesso, G. \& Zanoni, E., A Review on the Physical Mechanisms That Limit the Reliability of GaN-Based LEDs. IEEE Transactions on Electron Devices 57 (1), 108-118 (2010).

6. Kim, B.-J. et al., Buried graphene electrodes on GaN-based ultra-violet light-emitting diodes. Applied Physics Letters 101, 031108 (2012).

7. Hirayama, H., Maeda, N., Fujikawa, S. \& Toyoda, S. . K. N., ecent progress and future prospects of AIGaN-based high-efficiency deep-ultraviolet light-emitting diodes. Japanese Journal of Applied Physics 53 (10), 100209 (2014).

8. Chowdhury, A., Ng, H. M., Bhardwaj, M. \& Weimann, N. G., Second-harmonic genertion in periodically poled GaN. Applied Physics Letters 83, 1077-1079 (2003).

9. Hite, J. et al., Development of periodically oriented gallium nitride for non-linear optics. Optical Materials Express 2, 1203-1208 (2012).

10. Bowman, S. R. et al., Broadband measurements of the refractive indices of bulk Gallium Nitride. Optical Materials Express 4 (7), 1287-1296 (2014).

11. Morkoc, H. Handbook of Nitride Semiconductors and Devices, vol. 2, Wiley-VCH, Weinheim, 2008.

12. Liu, F. et al., The mechanism for polarity inversion of GaN via a thin AIN layer: Direct experimental evidence. Applied Physics Letters 91, 203115 (2007). 
13. Stutzmann, M. et al., Playing with Polarity. Physica Status Solidi B 228, 505-512 (2001).

14. Yoshikawa, A. \& Xu, K., Polarity selection process and polarity manipulation of GaN in MOVPE and RF-MBE growth. Thin Solid Films 412, 38-43 (2002).

15. Dimitrov, R. et al., Lateral polarity heterostructures by overgrowth of patterned AlxGa1-xN nucleation layers. Material Research Society Symposium 622, T4.6 (2000).

16. Hoffmann, M. P. et al., Growth and Characterization of AlxGa1-xN lateral polarity structures. Physica Status Solidi A 212 (5), 1039-1042 (2015).

17. Kirste, R. et al., Polarity Control and growth of lateral polarity structures in AIN. Applied Physics Letters 102, 181913 (2013).

18. Romano, L. T., Northrup, J. E., Ptak, A. J. \& Myers, T. H., Faceted inversion domain boundary in GaN films doped with Mg. Applied Physics Letters 77 (16), 2479-2481 (2000).

19. Komninou, P. et al., Interfacial and defect structures in multilayered GaN/AIN films. Journal of Physics: Condensed Matter 14, 13277-13283 (2002).

20. Lee, J. M., Chang, K. M, Kim, S. W., Huh, C., Lee, I. H., Park, S. J., Dry etch damage in n-type GaN and its recovery by treatment with an N2 plasma. Journal of Applied Physics 87 (11), 7667-7670 (2000).

21. Cao, X. A., Pearton, S. J., Dang, G. T., Zhang, A. P., Ren, F., Van Hove, J. M., GaN N- and Ptype Schottky diodes: effect of etch damage. IEEE Transactions on Electron Devices 47 (7) 1320-1324 (2000).

22. Eddy Jr., C. R., Molnar, B., Plasma etch-induced conduction changes in gallium nitride. Journal of Electronic Materials 28 (3), 314-318 (1999).

23. Hite, J. K. et al., GaN vertical and lateral polarity heterostructures on GaN substrates. Journal of Crystal Growth 332, 43-47 (2011).

24. Eddy, Jr., C. R., Nepal, N., Hite, J. K., Mastro, M. A. Perspectives on Future Directions in III-N Semiconductor Research. Journal Vacuum Science and Technology A 31 (5), 058501 (2013).

25. Hite, J. K., Twigg, M. E., Mastro, M. A., Eddy Jr., C. R. \& Kub, F. J., Initiating polarity inversion in GaN growth using an AIN interlayer. Physica Status Solidi A 208, 1504-1506 (2011).

26. Kirste, R. et al., Properties of AIN based lateral polarity structures. Physica Status Solidi C 11 (2), 261-264 (2014).

27. Hite, J. K., Zoll, R., Mastro, M. M. \& Eddy Jr., C. R., Role of growth parameters in equalizing simultaneous growth of N- and Ga-polar GaN by MOCVD. Physica Status Solidi C 11 (3-4), 458-461 (2014).

28. Ng, H. M., Parz, W. \& Weimann, N. G. . C. A., Patterning GaN Microstructures by PolaritySelective Chemical Etching. Japanese Journal of Applied Physics 42 (12A), L1405-L1407 (2003).

29. Katayama, R., Kuge, Y., Onabe, K., Matsushita, T. \& Kondo, T., Complementary analyses on the local polarity in lateral polarity-inverted GaN heterostructure on sapphire (0001) substrate. Applied Physics Letters 89, 231910 (2006).

30. Matsumura, H. et al., Lateral Polarity Control in GaN Based on Selective Growth Procedure Using Carbon Mask Layers. Applied Physics Express 2, 101001 (2009).

31. Mita, S., Collazo, R. \& Sitar, Z., Fabrication of a GaN lateral polarity junction by metalorganic 
chemical vapor deposition. Journal of Crystal Growth 311, 3044-3048 (2009).

32. Kirste, R. et al., Temperature dependent photoluminescence of lateral polarity junctions of metal organic chemical vapor deposition grown GaN. Journal of Applied Physics 110, 093503 (2011).

33. Park, M. et al., Micro-Raman study of electronic properties of inversion domains in GaNbased lateral polarity heterostructures. Journal of Applied Physics 93 (12), 9542-9547 (2003).

34. Fichtenbaum, N. A., Mates, T. E., Keller, S., DenBaars, S. P. \& Mishra, U. K., Impurity incorporation in heteroepitaxial N-face and Ga-face GaN films grown by metalorganic chemical vapor deposition. Journal of Crystal Growth 310, 1124-1131 (2008).

35. Yang, W. -C. et al., Photoelectron emission microscopy observation of inversion domain boundaries of GaN-based lateral polarity heterostructures. Journal of Applied Physics 94 (9), 5720-5725 (2003).

36. Niebelschutz, M., Ecke, G., Cimalla, V., Tonisch, K. \& Ambacher, O., Work function analysis of GaN-based lateral polarity structures by Auger electron energy measurements. Journal of Applied Physics 100, 074909 (2006).

37. Schuck, P. J. et al., Spatially resolved photoluminescence of inversion domain boundaries in GaN-based lateral polarity heterostructures. Applied Physics Letters 79 (7), 952-954 (2001).

38. Fiorentini, V., Origin of the efficient light emission from inversion domain boundaries in GaN. Applied Physics Letters 82, 1182-1184 (2003).

39. Liu, F. et al., Direct Observation of Inversion Domain Boundaries of GaN on c-Sapphire at Sub-Angstrom Resolution. Advanced Materials 20, 2162-2165 (2008).

40. Collaxo, R., Mita, S., Rice, A., Dalmau, R. F. \& Sitar, Z., Simultaneous growth of a GaN p/n lateral polarity junction by polarselective doping. Applied Physics Letters 91, 212103 (2007).

41. Collazo, R. et al., Implementation of the GaN lateral polarity junction in a MESFET utilizing polar doping selectivity. Physica Status Solidi A 207 (1), 45-48 (2010).

42. Hite, J. K. et al., Selective switching of GaN polarity on Ga-polar GaN using atomic layer deposited Al2O3. Applied Physics Express 7, 025502 (2014).

43. Song, J., Yuan, G., Xiong, K., Leung, B. \& Han, J., Epitaxial Lateral Overgrowth of NitrogenPolar (000I) GaN by Metalorganic Chemical Vapor Deposition. Crystal Growth and Design 14 (5), 2510-2515 (2014).

44. Wong, M. H. et al., N-polar GaN epitaxy and high electron mobility transistors. Semiconductor Science and Technology 28 (7), 074009 (2013).

45. Pezzagna, S., Vennegues, P., Grandjean, N., Wieck, A. D. \& Massies, J., Submicron periodic poling and chemical patterning of GaN. Applied Physics Letters 87, 062106 (2005).

46. Ferguson, J. D. et al., Determination of GaN polarity on periodically oriented surfaces. Journal of Vacuum Science \& Technology B 33, 011206 (2015).

47. Brown, C. G. et al., Frequency conversion in free-standing periodically oriented Gallium Nitride. Proceedings of the SPIE 9731, 97310E (2016). 


\section{Figure Captions}

Figure 1. Process schematic to create periodically oriented III-nitride materials.

Figure 2. TEM cross-section of PO GaN structure on an N-polar GaN template. New dislocations initiating at inversion layer interface in Ga-polar region can be observed.

Figure 3. Cross-sectional SEM image of a thick $(80 \mathrm{~mm})$ PO GaN structure grown by HVPE on a 2 $\mu \mathrm{m}$ thick MOCVD-grown PO GaN template on a bulk HVPE substrate. The saw-tooth pattern at the surface is formed by differences in growth rate between the two polar regions. 
